\title{
Effects of Exercise Using a Stick on Muscle Activity of Gluteus Maximus and Erector Spinae during Backward Return from Forward Bending
}

\author{
Won-hwee Lee, PT, Ph.D \\ Department of Physical Therapy, Vision College of Jeonju, Jeonju, South Korea.
}

Background Chronic lower back pain patients commonly present with impaired lumbar movement patterns, which may result in pain and excessive loads on the lumbar spine. A stick exercise can be effective to correct impaired movement patterns during forward bending in patients with lumbar flexion syndrome.

Purpose This study investigated the effect of exercise using a stick on muscle activity of gluteus maximus and erector spinae during backward return from forward bending.

Study design Comparative, repeated measures design.

Methods A total of 25 male patients with lumbar extension syndrome were recruited to this study. All patients performed backward return from forward bending with and without a stick. Gluteus maximus and erector spinae surface electromyography activities during backward return from forward bending were measured.

Results There was a significant difference in gluteus maximus and erector spinae muscle activities, on both sides, with versus without use of the stick. During backward return from forward bending with a stick, the activities of the right and left gluteus maximus increased significantly $(p<0.05)$, and those of the right and left erector spinae decreased significantly $(p<0.05)$, compared to without a stick.

Conclusions This study suggests that use of a stick can minimize excessive lumbar extension during backward return from forward bending.

Key words Backward return from forward bending; Erector spinae; Gluteus maximus; Lower JMST

\section{CONTACT}

whlee@jvision.ac.kr Won-hwee Lee, Department of Physical Therapy, Vision College of Jeonju, South Korea

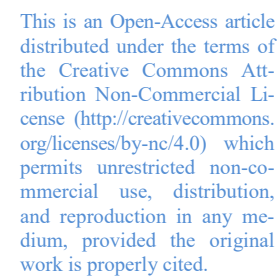
back pain; Stick exercise.

\section{INTRODUCTION}

Lower back pain (LBP) is often recurrent and is considered a multifactorial condition. The most common form of lower back pain is non-specific LBP. ${ }^{1-2}$ Chronic nonspecific LBP is commonly observed in people aged $<45$ years in the United States. ${ }^{3}$ Furthermore, LBP is the second most common reason for clinical visits and the fifth most common reason for hospital admission for surgical procedures. ${ }^{4}$

Chronic LBP commonly presents with limited range and velocity of lumbar movements, and abnormal trunk muscle contraction. ${ }^{5}$ Aberrant trunk movement patterns can lead to excessive load on the lumbar spine and could be a predis- posing factor for recurrent LBP episodes. ${ }^{6}$ Sahrmann $^{7}$ suggested that spinal dysfunction is commonly the result of cumulative microtrauma caused by impairments in the alignment, stabilization, and movement patterns of the spine.

Esola et al. ${ }^{8}$ reported differences in lumbar-hip movement patterns during forward bending between individuals with and without a history of LBP. During backward return, lumbopelvic extension to an upright position from a forward, flexed position is initiated at the hip. Contributions from the lumbar spine and hip during the middle phases of extension are relatively equal, whereas during the final phase of extension, the lumbar spine contributes the most movement. ${ }^{6}$ Paquet et al. ${ }^{9}$ found that participants with 
subacute LBP showed greater erector spinae activity at the end of flexion and the beginning of extension compared to healthy participants, who showed minimal erector spinae activity at these times. Sahrmann ${ }^{7}$ also reported that patients with LBP initiate motion at the lumbar spine immediately after a short period of hip extension. LBP patients with lumbar extension syndrome, as determined by the classification of Sahrmann, showed more symptoms when leading with lumbar extension or exhibiting forward sway of the pelvis and posterior sway of the thorax during the return from forward bending, ${ }^{7}$ possibly due to greater recruitment of the back extensor muscles than the hip extensors. Symptoms should decrease by extending the hips and only gradually extending the back. ${ }^{7}$

Most studies have shown a difference in movement patterns between those with LBP and healthy individuals. ${ }^{10-12}$ Some studies have explored how to correct dysfunctional movement patterns in LBP. ${ }^{13-14}$ Choung et al. ${ }^{13}$ and Yoon et al. ${ }^{14}$ suggested that exercises using a stick were effective for correcting impaired movement patterns during forward bending in patients with lumbar flexion syndrome. However, no studies have explored how to correct impaired movement patterns in patients with lumbar extension syndrome. Therefore, we investigated whether use of a stick to correct backward return from forward bending affects hip and back extensor activities. The gluteus maximus muscle is the major hip extensor and the primary action of the erector spinae muscles is lumbar extension. ${ }^{15}$ Thus, this study investigated the effect of exercise using a stick on muscle activity of gluteus maximus and erector spinae, during backward return from forward bending.

\section{METHODS}

\section{Participants}

Twenty-five male patients with lumbar extension syndrome (mean age, 22.9 \pm 1.8 years; mean height, $1.74 \pm 0.05 \mathrm{~m}$; mean weight, $71.97 \pm 11.76 \mathrm{~kg}$ ) were recruited to this study. Participants with lumbar extension syndrome were classified based on the criteria of Van Dillen et al. ${ }^{16}$ Lumbar extension syndrome can be assessed according to alignment during the return from forward bending in a standing position, alignment and hip and knee flexion in a supine position, alignment and knee flexion and hip extension in a prone position, and alignment and trunk extension in a sitting position. Participants were diagnosed with lumbar extension syndrome if the lumbar spine tended to move in the direction of extension, and if the lumbar spine tended to be extended with respect to the neutral position, symptoms increased when the lumbar spine was in extension, and symptoms decreased with restriction of lumbar extension. The inclusion criteria were that each participants were classified lumbar extension syndrome and able to backward return from forward bending in a standing position. The exclusion criteria were lumbar spine or hip joint contracture, and significant weakness of the trunk extensors that would interfere with the return from forward bending in a standing position. Approval for the study was granted by the Korea National Institute. For Bioethics Policy and all participants provided written informed consent prior to testing.

\section{Instrumentation}

Electromyography (EMG) data were collected using the BTS FreeEMG100RT instrument (BTS Bioengineering, Italy) and analyzed using EMG Analyzer software (BTS Bioengineering). A digital band-pass filter was used to filter movement artifacts $(20-500 \mathrm{~Hz})$. The sampling rate was set at 1,000 Hz. The EMG signals were processed to obtain root mean square values using a moving 50-ms window. Electrodes were placed on the gluteus maximus (between the greater trochanter and the sacral vertebrae in the middle of the muscle, at an oblique angle at the level of the greater trochanter or slightly above) and the erector spinae (parallel to and approximately $2 \mathrm{~cm}$ from the spine over the muscle belly) bilaterally. ${ }^{17}$ To minimize skin resistance, these sites were shaved and cleaned with rubbing alcohol. For normalization, maximum voluntary isometric contraction (MVIC) was measured for the gluteus maximus and erector spinae muscles. The measurement locations for MVIC were based on the manual muscle testing procedures recommended by Kendall. ${ }^{18}$ The examiner asked each participant to perform a 5-s MVIC three times in each position, with a 1-minute rest period between three trials to avoid muscle fatigue. The average EMG activity was expressed as a percentage of the MVIC value (\%MVIC).

\section{Procedure}

Participants performed backward return from the forward bending position with and without lumbar extension. They were instructed to stand in a forward-bending posture, via hip flexion without lumbar movement until the hip joint was flexed by 30 degrees (without bending the knee). In the first condition (without stick), participants were instructed to stand in the forward bending posture while facing backwards, and to return to the upright standing position for $3 \mathrm{~s}$. Participants performed the backward return movement. To control the pace of backward return, we used a metronome. In the second condition, participants performed backward return with a stick; lumbar extension was limited using a 1- 
m-long stick ( $2 \mathrm{~cm}$ in diameter). Participants held the stick against the occiput, with the other hand on the sacrum, and performed backward return to keep the thoracic and lumbar spine in contact with the stick through contraction of the abdominal muscles (Figure 1). Choung et al. ${ }^{13}$ suggested that forward bending with a stick is more effective than manual facilitation in terms of inducing changes in lumbar spine and hip joint angles. Yoon et al. ${ }^{14}$ reported that an exercise using a stick was effective to prevent excessive lumbar motion and increased hip motion during forward-bending.

Our participants performed three trials under each condition; the order of the two conditions was randomized. EMG data were collected over $3 \mathrm{~s}$ during the three trials in each condition. The participants were allowed to rest for 1 min between trials and there was a 5-min gap between two conditions to minimize muscle fatigue.

\section{Statistical analysis}

A paired $t$-test was used to compare changes in the activity of each muscle between the two conditions. The significance level was set at 0.05. SPSS for Windows software (ver. 19.0; SPSS, Inc., Chicago, IL, USA) was used for the statistical analyses.

\section{RESULTS}

There was a significant difference between the two conditions with respect to the activities of the gluteus maximus and erector spinae muscles on both sides. When performing backward return from forward bending in the stick condition, the activities of the right and left gluteus maximus increased significantly $(p<0.05)$, and those of the right and left erector spinae decreased significantly $(p<0.05)$, compared to without a stick (Table 1 and Figure 2).

\section{DISCUSSION}

Clinicians use forward bending, and backward return from forward bending, as part of routine clinical examinations for evaluating spine mobility, and to identify movement impairments of the lumbar spine, such as lumbar flexion syndrome and extension syndrome., ${ }^{7,19}$ Backward return from forward bending represents an organized movement pattern characterized by coordination of the lumbar spine and hip (in connection with the pelvis) in the sagittal plane. Altered movement patterns of the spine and hip may contribute to the development of LBP. ${ }^{20}$ LBP patients with lumbar extension syndrome exhibit excessive lumbar extension during backward return from forward bending and show muscle and recruitment pattern dysfunction. ${ }^{7}$ The activity of the back extensor muscles was more dominant than of the hip extensors during backward return from forward bending. ${ }^{7}$ Many clinicians have recommended using a stick to prevent unwanted excessive lumbar movement. However, no study has investigated the

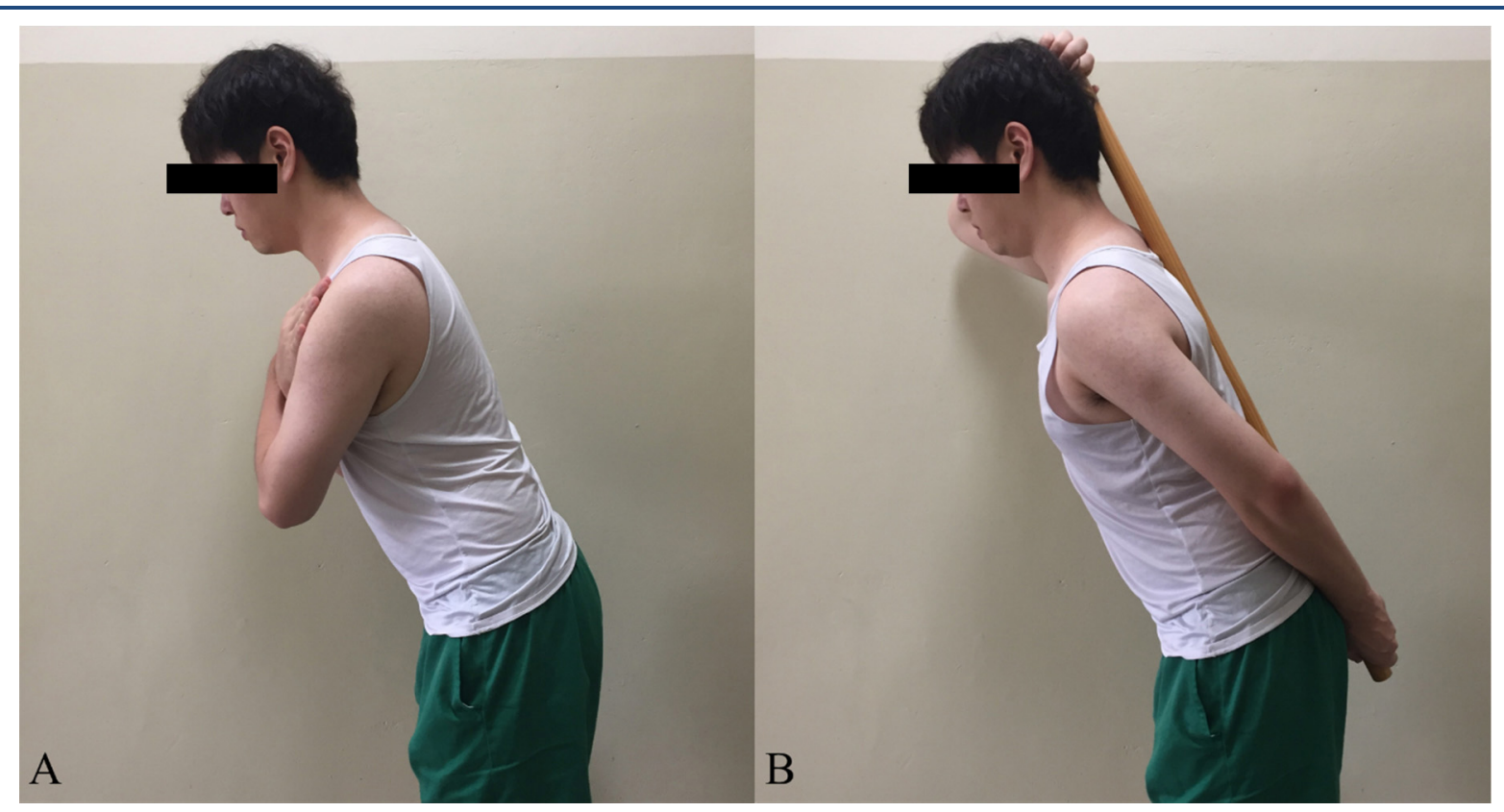

Figure 1. Experimental tasks (A. Backward return from forward bending without a stick, B. Backward return from forward bending with a stick). 
Table 1. Comparison of muscle activities between the exercises performed with and without a stick during backward return from forward bending

\begin{tabular}{ccccc}
\hline Muscles & $\begin{array}{c}\text { Without stick } \\
(\% \mathrm{MVIC})\end{array}$ & $\begin{array}{c}\text { With stick } \\
(\% \mathrm{MVIC})\end{array}$ & $t$ & $p$ \\
\hline Right gluteus maximus & $10.95 \pm 4.91$ & $16.37 \pm 10.68$ & -2.66 & $0.01^{*}$ \\
\hline Left gluteus maximus & $9.86 \pm 9.88$ & $16.03 \pm 14.23$ & -2.85 & $0.01^{*}$ \\
\hline Right erector spinae & $44.27 \pm 14.93$ & $39.04 \pm 14.83$ & 2.88 & $0.01^{*}$ \\
\hline Left erector spinae & $49.31 \pm 13.24$ & $42.30 \pm 11.72$ & 2.77 & $0.01^{*}$ \\
\hline
\end{tabular}

Abbreviations: MVIC, maximal voluntary isometric contraction.

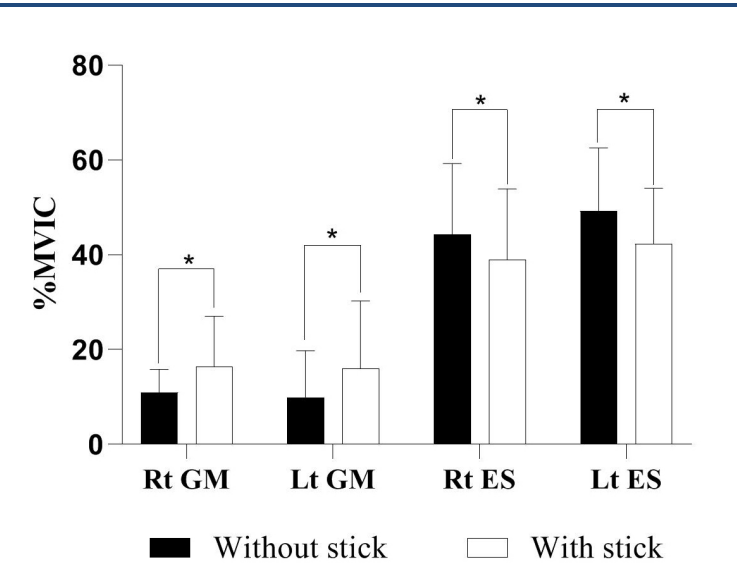

Figure 2. Comparison of muscle activities between the exercises performed with and without a stick during backward return from forward bending.

Abbreviations: Rt GM, right gluteus maximus; Lt GM, Left gluteus maximus; Rt ES, right erector spinae; Lt ES, left erector spinae; MVIC, maximal voluntary isometric contraction.

effects of exercises using a stick on lumbar and hip movement during backward return from forward bending.

In this study, lumbar and hip extensor activity changed significantly during backward return from forward bending under the stick condition; when participants performed backward return from forward bending, the gluteus maximus activity, which extended the hip joint, increased significantly, while that of the erector spinae, which extended the lumbar spine, decreased significantly compared to without a stick. Thus, the return from forward bending showed limited lumbar movement with use of the stick. Choung et al. ${ }^{13}$ and Yoon et al. ${ }^{14}$ suggested that sticks can effectively prevent excessive lumbar motion during the forward-bending exercise. Although this study did not measure lumbar or hip motion, significant increases in gluteus maximus activity, and decreases in erector spinae activity, in our LBP patients with lumbar extension syndrome suggests that a stick can minimize excessive lumbar extension during backward return from forward bending.

There are several possible explanations for the limited excessive lumbar extension seen in the participants when performing backward return from forward bending under the stick condition. First, the participants maintained contact of their thoracic and lumbar spine with the stick by contraction of their abdominal muscles. Thus, the tautness of the abdominal muscles and the stick limited excessive lumbar extension. Second, tactile feedback from the stick allowed the participants to limit excessive lumbar extension during backward return from forward bending. Choung et al. ${ }^{13}$ reported that forward bending with use of a stick provided a larger contact surface with the spine and greater proprioceptive feedback versus manually facilitated forward bending, thus more effectively restricting lumbar motion and enhancing hip motion. Therefore, use of a stick during backward return from forward bending should be considered to change movement patterns in patients with lumbar extension syndrome.

Delayed recruitment or weak activation of the gluteus maximus induces compensatory overload of the lumbar spine and simultaneous overactivity of the thoracolumbar erector spinae. ${ }^{21}$ Lewis et al. ${ }^{22}$ reported that patients with anterior hip pain showed delayed onset of the gluteus maximus. Thus, the gluteus maximus is an important muscle for pelvic stability. ${ }^{21}$ To strengthen the gluteus maximus, various exercises are available, such as seated gluteus maximus squeezing, sit-to-stand, bridging exercises, and prone hip extension with a flexed knee. ${ }^{23}$ The stick exercise in this study may also help activate and recruit a weak gluteus maximus.

Assessment of movement patterns may help identify differences between individuals with and those without a history of LBP, which could lead to more specific and improved treatment strategies aimed at regaining normal motion patterns. If altered patterns of motion were present in individuals after an episode of LBP, rehabilitation could 
be directed at improving faulty motion patterns, which may decrease recurrence. ${ }^{6}$ Therefore, studies related to exercise of correction of altered movement patterns in LBP should be continued.

This study had several limitations. First, it did not directly measure the lumbar or hip extension, nor the onset time of gluteus maximus and erector spinae activity. Moreover, muscle activity of the hamstring muscle was not assessed. The hamstring muscle can affect lumbar and hip motion during backward return from forward bending; further studies are required to measure the lumbar and hip extension angle during this movement. Muscle activity of the hamstring and onset time of gluteus maximus and erector spinae activities should also be measured during backward return from forward bending movement.

\section{CONCLUSIONS}

This study investigated the effect of exercise using a stick on muscle activity of gluteus maximus and erector spinae in lumbar extension syndrome during backward return from forward bending. When participants returned from forward bending under the stick condition, gluteus maximus activity increased significantly, and erector spinae activity decreased significantly, compared to without a stick. Therefore, use of a stick during backward return from forward bending should be considered to improve movement patterns in patients with lumbar extension syndrome.

\section{Key Points}

Question Can a stick exercise affect gluteus maximus and erector spinae activities during backward return from forward bending?

Findings During backward return from forward bending, the activities of the right and left gluteus maximus increased significantly, and those of the right and left erector spinae decreased significantly, with versus without a stick.

Meaning This study suggests that use of a stick can minimize excessive lumbar extension during backward return from forward bending.

\section{Article information}

Conflict of Interest Disclosures: None.

Funding/Support: None.

Acknowledgment: None.

Ethic Approval: This study received the approval of the
Korea National Institute.

\section{REFERENCES}

1. Balagué F, Mannion AF, Pellisé F, et al. Non-specific low back pain. Lancet. 2012;379(9814):482-491.

2. Maher C, Underwood M, Buchbinder R. Non-specific low back pain. Lancet. 2017;389(10070):736-747.

3. Lin ML, Wu JH, Lin CW, et al. Clinical effects of laser acupuncture plus chinese cupping on the pain and plasma cortisol levels in patients with chronic nonspecific lower back pain: a randomized controlled trial. Evid Based Complement Alternat Med. 2017;2017:3140403.

4. Ladeira CE. Evidence based practice guidelines for management of low back pain: physical therapy implications. Rev Bras Fisioter. 2011;15(3):190-199.

5. van Dieen JH, Reeves NP, Kawchuk G et al. Motor control changes in low-back pain: divergence in presentations and mechanisms. J Orthop Sports Phys Therapy. 2019;49(6):370-379.

6. McClure PW, Esola M, Schreier R et al. Kinematic analysis of lumbar and hip motion while rising from a forward, flexed position in patients with and without a history of low back pain. Spine (Phila Pa 1976). 1997;22(5):552-558.

7. Sahrmann SA. Diagnosis and treatment of movement impairment syndrome. New York. USA, Mosby. 2002.

8. Esola MA, McClure PW, Fitzgerald GK, et al. Analysis of lumbar spine and hip motion during forward bending in subjects with and without a history of low back pain. Spine (Phila Pa 1976). 1996;21(1):71-78.

9. Paquet N, Malouin F, Richards CL. Hip-spine movement interaction and muscle activation patterns during sagittal trunk movements in low back pain patients. Spine (Phila Pa 1976). 1994;19(5):596-603.

10. Marich AV, Hwang CT, Salsich GB, et al. Consistency of a lumbar movement pattern across functional activities in people with low back pain. Clin Biomech (Bristol, Avon). 2017;44:45-51.

11. Hernandez A, Gross K, Gombatto S. Differences in lumbar spine and lower extremity kinematics during a step down functional task in people with and people without low back pain. Clin Biomech (Bristol, Avon). 2017;47:46-52.

12. Scholtes SA, Gombatto SP, Van Dillen LR. Differences in lumbopelvic motion between people with and people without low back pain during two lower limb movement tests. Clin Biomech (Bristol, Avon). 2009;24(1):7-12.

13. Choung SD, Park KN, Hong JA, et al. Change of lumbar spine and hip joint flexion angles during 
forward bending of the trunk using manual facilitation and a stick. Korean Research Society of Physical Therapy. 2011;18(1):57-63.

14. Yoon JY, Kim JW, Kang MH, et al. The effects of an exercise with a stick on the lumbar spine and hip movement patterns during forward bending in patients with lumbar flexion syndrome. J Back Musculoskelet Rehabil. 2015;28(2):359-364.

15. Blandine CG. Anatomy of movement revised ed. Pennington, United States: Princeton Book Company; 2008.

16. Van Dillen LR, Maluf KS, Sahrmann SA. Further examination of modifying patient-preferred movement and alignment strategies in patients with low back pain during symptomatic tests. Man Ther. 2009;14(1):52-60.

17. Cram J, Kasman G, Holtz J. Introduction of surface electromyography. Gaithersburg. MD: Aspen Publishers, 1988.

18. Kendall FP, McCreary EK, Provance PG, et al. Muscles: testing and function, wirh postural and pain. $5^{\text {th }}$.
Baltimore: Lippincott Williams \& Wilkins; 2005.

19. Shojaei L, Vazirian M, Salt EG, et al. Timing and magnitude of lumbar spine contribution to trunk forward bending and backward return in patients with acute low back pain. $J$ Biomech. 2017;53:71-77.

20. Zawadka M, Skublewska-Paszkowska M, Gawda P, et al. What factors can affect lumbopelvic flexionextension motion in the sagittal plane?: a literature review. Hum Mov Sci. 2018;58:205-218.

21. Page P, Frank CC, Lardner R. Assessment and treatment of muscle imbalance. The janda approach. Champaign: Human Kinetics; 2010.

22. Lewis CL, Sahrmann SA, Moran DW. Anterior hip joint force increases with hip extension, decreased gluteal force, or decreased iliopsoas force. J Biomech. 2007; 40(16):3725-3731.

23. Lee D, Hodges P. The pelvic girdle. An approach to the examination and treatment of the lumbopelvic-hip region. $3^{\text {rd }}$ ed. Churchill Livingstone; 2004. 